\title{
Ethical aspects of plans to combat Huntington's disease-
}

\section{a response}

\author{
Michael H Kottow Charlottenklinik for Augenkranke, Stuttgart, West Germany
}

\section{Editor's note}

In response to the late Dr Brackenridge's article (I) on some dilemmas posed by plans to combat Huntington's disease DrKottow discusses three issues which any proposal to introduce a new way of combatting disease must address. First, how good is the research and the rationale underpinning the new proposal? Research indicates that the proposed use of levodopa provocation to detect Huntington's would be inefficient and potentially dangerous. Second, how can the developing knowledge be beneficially applied in practice? The information available suggests that in practice no reasonable options offering more benefit than harm could be offered to potential sufferers. Third, who should make the choice, once reasonable options do become available? DrKottow argues that this should be left to the patient once information has been passed to him 'in a tactful and humane manner'.

Dr Brackenridge's article 'Ethical aspects of plans to combat Huntington's disease' ( $I$ ) is a perceptive and most timely analysis, appearing in a period characterised by rapid knowledge turnover plus growing ethical inquisitiveness. The problems alluded to in the paper can be subsumed under the more general aspect of ethical issues related to unestablished medical practices, and appear in need of further discussion.

There is a tendency to leave hanging the various issues contained in the problems of introducing, maintaining or demoting experimental or insufficiently proven therapies. In this sense, Dr Brackenridge's article fails to stress that tentative plans to combat Huntington's or any other disease, must first address at least three issues:

I) Weighing the quality of research and medical thinking supporting the empirical basis of the plans;

2) Application of developing knowledge;

3) Assessment of anticipated results and consequences.

\section{Quality of research and medical thinking}

Research is of course value-laden and therefore amenable to ethical analysis. Ethical decisions are involved in allocation of resources, the choice of problems to be tackled and the design of experiments. Even the kind of questions an investigator asks reflects his ethical stance. But the problem at this point is not this ethical component of the basics of medical research. Rather, it involves the fact that data being accumulated by research efforts naturally needs to be calibrated as to consisten $\vec{\Phi}$ and truth content before this information is further elaborated or made free for clinical application.

In addition to analysing the empirical daf; underlying and resultant medical thinking neeकs also to be evaluated. The importance of cleat thinking as a correlate to tidy researching is show in the multiple examples of serendipitious dif coveries.

In his introduction, Dr Brackenridge presents the state of the art of predicting, diagnosing and managing Huntington's disease. He leaves no douBt about the fact that he finds available data insuff cient and contradictory, but he is willing to discus ethical issues on the basis of these inconclusive data. For example, provocative tests with levodop $\overrightarrow{3}$ which have an efficiency no higher than 50 per @erff, may be harmful to those responding positively Under these circumstances, it is not an etrigal decision to avoid mass-screening programmes, it is rather a matter of sound medical judgement. In similar fashion, if delayed-onset manageme $\mathrm{pt}$ will be unnecessary for half the treated population whilst possibly aggravating the disease in those affected, it seems obvious that one is not facing am ethical dilemma, but forgetting to analyse the harri: benefit ratio of a medical action.

\section{Application of developing knowledge}

Developing knowledge and technology face the practising physician with the everyday dilemra of deciding when, to whom and to what extent insufficiently proven diagnostic procedures or treatments should be offered. It would be quife simple to state that all available therapies must indiscriminately offered and the patient left decide. The problem is that for developing technology enough data for informed decisions ju are not available, so that one would be confrontifis patients with excessively diffuse and unbearab?y uncertain outcomes. This is especially true new diagnostic procedures of unknown risk asd uncertain specificity, especially if they cannot be followed by efficient therapy.

When contemplating the advantages of informed decision one must constantly bear in mind that one is dealing with a person under physical amd 
mental duress and that even if information is sufficiently available and clearly perceived (2), it introduces a new knowledge status in the patient which he may have difficulties in coping with. Take a simplified form of chorea counselling based on the data offered in Brackenridge's paper:

You are the apparently healthy young daughter of a chorea patient and have a 50 per cent chance of developing the disease. We might try and provoke it with levodopa. If you don't react, we still won't know if you are affected. If you do react, we will be sure you have chorea, but may have aggravated or anticipated the clinical manifestations of the disease. Furthermore, whatever the result, it will be advisable that you should avoid becoming pregnant and not pursue your stressful career. The same recommendations are valid if you don't take the test. On the other hand, should you take the test and be found to be diseased, we may be harming you by suggesting you avoid stress and pregnancy, since this might lead to a delayed but more severe form of chorea or, potentially worse still, you might become deranged or criminal without anyone recognising this to be due to chorea.

This is fair, but probably damaging information, and illustrates that inconclusive medical knowledge is unlikely to lead to reasonable options. In addition, it must be remembered that both medical researchers and clinicians have been shown to be poor assessors of the variables involved in clinical judgement, so that our ability to diagnose, predict and evaluate disease hardly qualifies us to influence other people's decisions, especially if the knowledge involved is fluctuating (3).

Research results can only enter the realm of medical practice when they carry clear chances of offering more advantages than risks. Diagnostic procedures must be more informative than risky and be of potential use in management and therapy. Treatments must offer calculable risks and expected benefits must outweigh complications and failure rates. High risk procedures are of course acceptable, provided the benefits obtainable are highly enough prized. Such decisions certainly fall in the realm of informed decision, but information flow between health counsellor and recipent can only obtain if the counsellor can reasonably well map-out the alternative possibilities of a course of action.

\section{Assessment of anticipated results and consequences}

When all is said and done, the health provider needs to develop some kind of strategy when facing a diseased person. In the case of chorea, the alternatives at present are to remain passive and provide symptomatic relief, to prolong life at the cost of ever-increasing severity of the disease, or to intend control over the disease by promoting a mild form of it.
This, contends Dr Brackenridge, is the dilemma of the physician. In fact, the physician's dilemma is long over, since therapeutic decisions should be taken by the patient or the person directly responsible for him when he is incapable of choice.

Needless to say, informed decision-a term preferable to the paternalistic informed consentis fraught with difficulties and can rarely be carried out to the fullest extent. It has been stressed that anguished, pained, mentally distressed and economically depressed patients may constitute poor candidates for full disclosure, especially if irreversible damage or progressive deterioration must be conveyed (4). In medical practice one occasionally sees physicians inflicting extremely insensitive forms of disclosure but, on the whole, insufficient information and gross manipulation of patients' opinion and choice are more prevalent.

Provided information is passed on in a tactful and humane manner-and I would consider it bad faith to dismiss this kind of approach by questioning what tactful and humane mean-and provided the physician does not forget to use to the utmost his ability and his diagnostic acumen to gauge the extent and timeliness of knowledge transfer, informed decision remains a fundamental goal of medical actions.

To summarise, immature medical technology should not become available as long as it has not reached a level of reliability compatible with informed prediction and likely expectations. It should be available only when a risk/benefit analysis clearly shows a positive balance, lest an anxious and unstable patient be led to take choices between nebulous or harmful alternative courses of medical action. Once medical options do become available, all decisions concerning their application should be passed on to the patient, provided no greater harm than benefit will thus accrue.

\section{References}

(I) Brackenridge C J. Ethical aspects of plans to combat Huntington's disease. Fournal of medical ethics $1981 ; 7$ : 24-27.

(2) Ingelfinger F J. Informed (but uneducated) consent. New England journal of medicine 1972; 287: 465-466

(3) Scriven M. Clinical judgment. In $\mathrm{H}$ T Engelhardt Jnr, Spicker S F, Towers B eds. Clinical judgement: $A$ critical appraisal. Dordrecht, $\mathrm{D}$ Reidel Publishing Co, 1979: 3-16.

(4) Pellegrino E D. Toward a reconstruction of medical morality: The primacy of the act of profession and the fact of illness. Fournal of medicine and philosophy 1979; 4: 32-56.

\section{Postcript}

To discuss a man's ideas is to honour his interests and respect his thoughts. It is distressing to see dialogue disrupted by death.

MHK 